\title{
Prevalence of Ciprofloxacin Resistance among Gram-Negative Bacilli Isolated From Different Specimens at a General Hospital in Riyadh, Saudi Arabia
}

\author{
Hossain $\mathrm{MA}^{1}$, Mohal $\mathrm{S}^{2}$, Yusuf $\mathrm{MA}^{3}$, Islam $\mathrm{MS}^{4}$, Islam $\mathrm{MR}^{5}$, Roy $\mathrm{S}^{6}$
}

\begin{abstract}
Background: Resistance among the different classes of antimicrobials including fluoroquinolones has arisen in a multitude of bacterial species in the hospitals and in the community. Objective: The purpose of the present study was to measure the prevalence of ciprofloxacin resistance as well as to evaluate the susceptibility pattern of the isolates to various antibiotics. Methodology: This cross sectional study was carried out in the Department of Microbiology at a General Hospital in Riyadh, Saudi Arabia from July'2009 to December'2009. Different clinical specimens were collected and culture was done in the appropriate media. Antibiotic susceptibility testing was performed. Gram negative bacterial isolates with decreased susceptibility to ciprofloxacin were tested for minimum inhibitory concentration (MIC) by using the E-test. Results: A total of 1246 gram-negative bacilli (GNB) were isolated from different specimens of which $486(39.0 \%)$ isolates were resistant to ciprofloxacin. The MIC of these isolates ranged from 4 to $32 \mu \mathrm{g} / \mathrm{ml}$. Most of the Ciprofloxacin resistant isolates were from urinary tract infections. Conclusion: The prevalence of ciprofloxacin resistance in different specimens is high. [J Shaheed Suhrawardy Med Coll, 2013;5(1):7-10]
\end{abstract}

Key words: MIC, Gram-negative bacilli, fluoroquinolones, ciprofloxacin

Received: January 2013; Revised: March 2013; Accepted: April 2013

\section{Introduction}

There were major therapeutic advance of fluoroquinolones antimicrobial in 1980, because they have 100 fold greater activities than their parent compound Nalidixic acid ${ }^{1}$. Previously Nalidixic acid was used only for urinary tract infections and occasionally for shigellosis². The fluoroquinolones have a broad range of therapeutic indications and are given as prophylaxis like in veterinary medicine fluoroquinolones are used as treatment and metaphylaxis ${ }^{3,4}$. Early researchers thought that fluoroquinolones resistance was likely developed resistance to Escherichia coli mutants and are exceptionally difficult to select in vitro ${ }^{5}$. Plasmid-mediated quinolone resistance was remained unknown even after 30 years of Nalidixic acid usage. Nevertheless multi-national quinolone resistance emerges in Staphylococci species and Pseudomonas aeruginosa which are inherently less susceptible than Escherichia coli.
Fluoroquinolones resistance has emerged in Escherichia coli and other enterobactericeae ${ }^{6}$. The multiple mutations diminish the affinity of its topoisomerase II and IV targets in varying ways reduce permeability and up regulate efflux ${ }^{6,7}$. However, plsmid-mediated quinolone resistance has been reported $^{7}$.

Ciprofloxacin is a broad spectrum antibiotic which is active against both Gram-positive and Gram-negative bacteria and it belongs to the fluoroquinolones class ${ }^{8}$. Bacterial resistance is a growing therapeutic problem both in the community and in the hospitals, involving all the antibiotics including fluoroquinolones ${ }^{9}$. A decreased susceptibility to fluoroquinolones arises mainly due to single-step mutations in the gyrA and the parC genes, which encode the fluoroquinolones targets which is the topoisomerase enzymes ${ }^{7,9}$. Accumulation to multiple mutations in several genes confers increasing level

1. Dr. Mohammad Afzal Hossain, Associate Professor, Department of Microbiology, Z.H. Sikder Women's Medical College, Dhaka

2. Dr. Shirin Mohal, Professor, Department of Anatomy, Z.H. Sikder Women's Medical College, Dhaka

3. Dr. Md. Abdullah Yusuf, Lecturer, Department of Microbiology, Shaheed Suhrawardy Medical College, Dhaka

4. Dr. Md. Saiful Islam, Lecturer, Department of Microbiology, Shaheed Suhrawardy Medical College, Dhaka

5. Prof. Dr. Md. Rafiqul Islam, Professor, Department of Microbiology, Shaheed Suhrawardy Medical College, Dhaka

6. Dr. Sushmita Roy, Assistant Professor, Department of Microbiology, Enam Medical College, Dhaka

Correspondence: Dr. Mohammad Afzal Hossain, Associate Professor and Head, Department of Microbiology, Z.H. Sikder Women's Medical College, Monica Estate, Western side of Dhanmondi, Dhaka-1209, Bangladesh; E-mail: ksmclab@gmail.com; Cell No.: +880173268107

Conflict of interest: No conflict of interest

Financial Support: None

Contributions by authors: Dr. M.A Hossain has preformed the work. Dr. S Mohal \& Dr. MA. Hossain have written the article. Dr. M.A. Yusuf \& Dr. MS Islam have revicued and modificd the article. Prof. Dr. MR Islam \& Dr. S.Roy have proved the article. 
of resistance associated with clinical failure ${ }^{8}$. Even low level resistance can generate therapeutic failure. Ciprofloxacin resistance is also closely associated with multi-drug resistance, thus limiting the treatment options.

The purpose of the present study was to evaluate the susceptibility of Gram-negative bacteria to various antibiotics and to know the prevalence rate of ciprofloxacin resistance.

\section{Methodology}

This was a cross sectional study carried out in the Department of Bacteriology at Central Lab and Blood Bank in King Saud Medical Complex, Riyadh, Saudi Arabia from July' 2009 to December'2009 for a period of six (6) months. Different clinical specimens like pus, urine, sputum, blood, CSF, wound swab and body fluids were received in the Bacteriology Laboratory. Specimens were processed using different media according to the types of specimens. Commonly used media were MacConkey's agar, sheep blood agar, cystine lactose electrolyte deficient (CLED) agar and chocolate agar. Gram-negative bacilli were isolated. All isolates were identified by using standard biochemical kits, API 20E (Analytical Profile Index System, La Balme Les Grottes, France) and the fully automated analyzers (Phoenix, Microscan, USA). Vitek-II was also used for the identification as well as to see sensitivity pattern of the pathogens. Antibiotic sensitivity testing was performed mainly by using the fully automated analyzers (Phoenix, Microscan \& Vitek II); however, sometimes disc diffusion method was used on $85 \mathrm{~mm}$ Mueller-Hinton agar (Oxoid ltd.). The bacterial suspension that was prepared for antibiotic sensitivity testing on Mueller-Hinton Agar or for the fully automated analyzers (Phoenix, Microscan \& Vitek II) were adjusted to the recommended turbidities for all species. The antibiotics tested on each disc were ampicillin $(25 \mu \mathrm{g})$, amoxicillinclavulanic acid $(20 / 10 \mu \mathrm{g})$, trimethoprim-sulphomethoxazole $(1.25-23.75 \mu \mathrm{g})$, cephalothin $(30 \mu \mathrm{g})$, cefuroxime $(30 \mu \mathrm{g})$, cefotaxime $(30 \mu \mathrm{g})$, cefepime $(30 \mu \mathrm{g})$, ciprofloxacin $(5 \mu \mathrm{g})$, norfloxacin $(30 \mu \mathrm{g}$-for urinary isolates), nalidixic acid (30 $\mu \mathrm{g}$-for urinary isolates), gentamicin $(10 \mu \mathrm{g})$, amikacin $(30 \mu \mathrm{g})$, tazocin (Piperacillin+Tazobactam), imipenem $(30 \mu \mathrm{g})$. The Clinical Laboratory Standards Institute (CLSI) break points were used for interpretation of susceptibility patterns as sensitive and resistant. Isolates with resistance or decreased susceptibility to ciprofloxacin $(20 \mathrm{~mm})$ were recorded. The study design and protocol was approved by "Research \& Ethics Committee" of the institute. The resistance to ciprofloxacin was confirmed by obserning the at break point minimum inhibitory concentration (MIC in $\mu \mathrm{g} / \mathrm{ml}$ ) by using E-test strips and also by the fully automated analyzers (Phoenix, Microscan \& Vitek II). The isolates with MIC value $4 \mathrm{mg} / \mathrm{ml}$ were defined as resistant isolates, as outlined by CLSI guidelines ${ }^{11}$. Computer based statistical analysis were carried out. All data were recorded systematically in preformed data collection form (questionnaire) and qualitative data were expressed as frequency distribution and percentage. Statistical analysis was performed by using window based computer software devised with Statistical Packages for Social Sciences (SPSS-19) (SPSS Inc, Chicago, IL, USA).

\section{Results}

A total of 1246 gram-negative bacilli were isolated from different clinical specimens.

Table 1: Isolation Rate of bacteria from Different specimens $(\mathbf{n}=1246)$

\begin{tabular}{lcc}
\hline Isolated Bacteria & Frequency & Percentage \\
\hline Escherichia coli & 380 & 30.5 \\
Klebsiella pneumoniae & 307 & 24.6 \\
Pseudomonas aeruginosa & 280 & 22.5 \\
Proteus species & 155 & 12.4 \\
Acinetobacter species & 65 & 5.2 \\
Citrobacter species & 27 & 2.2 \\
Enterobacter species & 17 & 1.4 \\
Morganella morganii & 10 & 0.8 \\
Burkholderia cepacia & 05 & 0.4 \\
Total & $\mathbf{1 2 4 6}$ & $\mathbf{1 0 0 . 0}$ \\
\hline
\end{tabular}

Escherichia coli $(30.5 \%)$ was the most predominant isolate which was found among the Gram negative bacteria followed by Klebsiella pneumoniae (24.6\%), Pseudomonas aeruginosa (23.5\%), Proteus species (12.4\%), Acinetobacter species (6.2\%), Citrobacter species (2.2\%), Enterobacter species (1.8\%), Morganella morganii $(0.6 \%)$ and Burkholderia cepacia $(0.4 \%)$ (Table 1$)$.

Table 2: Antibiotic susceptibility pattern of isolates to various antibiotics $(n=1246)$

\begin{tabular}{lcc}
\hline Name of Antibiotic & Sensitive n(\%) & Resistant n(\%) \\
\hline Ampicillin & $199(16.0 \%)$ & $1047(84.0 \%)$ \\
Amikacin & $1009(81.0 \%)$ & $237(19.0 \%)$ \\
Amoxicillin-Clavulanic Acid & $374(30.0 \%)$ & $872(70.0 \%)$ \\
Cephalothin & $311(25.0 \%)$ & $935(75.0 \%)$ \\
Cefuroxime & $773(62.0 \%)$ & $473(38.0 \%)$ \\
Cefotaxime & $810(65.0 \%)$ & $436(35.0 \%)$ \\
Cefepime & $984(79.0 \%)$ & $262(21.0 \%)$ \\
Ciprofloxacin & $760(61.0 \%)$ & $486(39.0 \%)$ \\
Gentamicin & $835(67.0 \%)$ & $411(33.0 \%)$ \\
Imipenem & $1171(94.0 \%)$ & $75(06.0 \%)$ \\
Trimethoprim-Sulphamethoxazole & $473(38.0 \%)$ & $773(62.0 \%)$ \\
Tazocin (Piperacillin+Tazobactam) & $1159(93.0 \%)$ & $87(07.0 \%)$ \\
\hline
\end{tabular}

Out of 1246 Gram-negative bacilli, 486(39.0\%) isolates were resistant to ciprofloxacin. High rates of resistance were observed for ampicillin and cephalothin followed by amoxicillin-clavulanic acid, trimethoprim-sulphamethoxazole, cefuroxime, cefotaxime while low levels of resistance were observed for nitrofurantoin, nalidixic acid, amikacin and norfloxacin (Table 2). 
Table 3: MIC values of the resistant Gram-Negative Bacilli to Ciprofloxacin $(n=486)$

\begin{tabular}{lcc}
\hline $\begin{array}{l}\text { MIC } \\
\text { Concentration }\end{array}$ & Frequency & Percentage \\
\hline $4 \mu \mathrm{g} / \mathrm{ml}$ & 97 & 20.0 \\
$8 \mu \mathrm{g} / \mathrm{ml}$ & 58 & 12.0 \\
$16 \mu \mathrm{g} / \mathrm{ml}$ & 68 & 14.0 \\
$32 \mu \mathrm{g} / \mathrm{ml}$ & 73 & 15.0 \\
$64 \mu \mathrm{g} / \mathrm{ml}$ & 190 & 39.0 \\
\hline
\end{tabular}

The lowest level of resistance was observed for tazocin $(7.0 \%)$ followed by Imipenem $(6.0 \%)$. The resistance rate of Ciprofloxacin was $39.0 \%$. The MIC of Ciprofloxacin for these isolates ranged from 4 to $>32 \mathrm{mg} / \mathrm{ml}$ (Table 3). The isolated bacteria showed wide differences in their susceptibility to ciprofloxacin. A high rate of resistance to ciprofloxacin was observed among Acinetobacter species, Pseudomonas aeruginosa, Klebsiella pneumoniae and Proteus species followed by Escherichia coli.

\section{Discussion}

The rapidly rising rates of fluoroquinolones-resistant Escherichia coli in many parts of the world have been found due to the reduced susceptibility to the quinolone. The Surveillance Network database shows resistance trends in blood-stream isolates from 250 U.S. hospitals ${ }^{11}$. Escherichia coli was $1.8 \%$ in 1996 and $4.3 \%$ in 1999; Klebsiella pneumoniae was $7.1 \%$ in 1996 and $6.7 \%$ in 1999; Proteus mirabilis was $5.7 \%$ in 1996 and $12.7 \%$ in $1999^{11}$. Much higher rates are reported from Barcelona ${ }^{7}$, Spain $^{12}$ where $17.0 \%$ Escherichia coli were ciprofloxacin resistant isolated from community infections. In India up to $50.0 \%$ hospital Escherichia coli are reported resistant ${ }^{13}$. The Spanish study ${ }^{14}$ found quinolone-resistant Escherichia coli isolated from foods in a rate of $90.0 \%$ due to chicken feces. There is a small set of drugs commonly used to treat Pseudomonas aeruginosa infection, including ciprofloxacin, tobramycin, gentamicin, ceftazidime and imipenem. While Pseudomonas aeruginosa has developed various levels of resistance to each of these antibiotics and its response to ciprofloxacin is of particular interest because the drug is initially very effective $^{15}$; however, Pseudomonas aeruginosa rapidly acquires high level of resistance. In clinical isolates, approximately $30.0 \%$ of strains present high-level ciprofloxacin resistance ${ }^{12,14}$. The resistance rates for ciprofloxacin were $39.0 \%$ in this present study which is very similar to previous study. Most of the resistant isolates were obtained from UTI samples and the reason of that fluoroquinolones are preferred as the initial agents for empiric therapy in UTI due to their excellent activity against the pathogens which are commonly encountered in UTI. This emphasizes the importance of the re-assessment of the antibiotics which are used in the empiric treatment of UTIs. Most of the isolates from UTIs were susceptible to nitrofurantoin, nalidixic acid, amikacin and imipenem. This is in agreement with the finding of a study reported by other studies $^{14-15}$.

These data suggests that nitrofurantoin can still be successfully used in the treatment of UTI. The ciproloxacin resistance was also closely associated with multi-drug resistance. Hence, it severely limits the already restricted treatment options. The finding was in accordance with the finding of a study which was conducted by Paterson et $\mathrm{al}^{16}$. The high resistance pattern which was seen in this study was probably due to the inappropriate prescribing of antibiotics which is sometimes doing without culture and sensitivity tests. In addition lack of antibiotic policy and the poor infection control strategies are also responsible though the antibiotic history could not be properly elicited from the patients of this study.

Ciprofloxacin remains a potent antibiotic; however, the slow accumulation of resistant enterobacteriaceae is disturbing. Resistance is a class effect affecting all fluoroquinolones ${ }^{12}$. Ultimately, this resistance may be partly overcome by the efflux pumps that contribute to the resistance but this strategy is still several years from fruition ${ }^{6}$. In the interim, the best approach lies in the prudent use of fluoroquinolones in humans and animals coupled with an emphasis on preventing patient-to-patient spread of resistant strains ${ }^{7}$.

The antibiotic which showed maximum activity against most of the isolates was imipenem \& tazocin. Though carbapenems remain the final options for treating these infections, there is a possibility that the increasing use of carbapenems may lead to a rapid emergence of carbapenems resistance. In this study, the considerably MIC values for Ciprofloxacin reflect the scope of limited treatment options which are available for these resistant isolates and a need for the continuous evaluation of the commonly used antibiotics. Repeated surveillance, the formulation of an antibiotic policy, the prudent prescriptions of antibiotics as well as the recycling of antibiotics are the possible routes which can be used to curb the rapid emergence and the spread of these resistant. The considerably high MIC values for ciprofloxacin in this study reflected the extent of the treatment problems for these resistant isolates and a need for the continuous evaluation of the commonly used antibiotics.

\section{Conclusion}

In the conclusion the considerably MIC values for ciprofloxacin reflect the scope of limited treatment options which are available for these resistant isolates and a need for the continuous evaluation of the commonly used antibiotics. Commonly isolated bacteria are highly resistant to ciprofloxacin.

\section{References}

1. Hooper DC. Emerging mechanisms of fluoroquinolone resistance. Emerging Infectious Diseases 2001;7(2):337

2. Schaeffer AJ. The expanding role of fluoroquinolones. American J Medicine 2002;113(1A):45S-54S

3. Bauernfeind A, Petermaller C. In vitro activity of ciprofloxacin, norfloxacin and nalidixic acid. European Clin Microbiol 1983;2(2):111-5

4. Martanez-MartÃnez L, Pascual A, Jacoby GA. Quinolone resistance from a transferable plasmid. The Lancet 1998;351(9105):797-9

5. Smith JT. The mode of action of 4-quinolones and possible mechanisms of resistance. Journal of Antimicrobial Chemotherapy 1986;18(Suppl D):21-9 
6. Ericsson HM, Sherris JC. Antibiotic sensitivity testing. Report of an international collaborative study. Acta pathologica et microbiologica Scandinavica Section B: Microbiol Immunology 1971;217:Suppl 217:1

7. Ahmad S. Prevalence of Ciprofloxacin Resistance Among GramNegative Bacilli at a Specialist Hospital in Saudi Arabia. Bangladesh J Medical Science 2012;11(4):317-321

8. Drlica K, Zhao X. DNA gyrase, topoisomerase IV, and the 4-quinolones. Microbiology and molecular biology reviews 1997;61(3):377-92

9. El Astal Z. Increasing ciprofloxacin resistance among prevalent urinary tract bacterial isolates in Gaza Strip, Palestine. BioMed Research International 2005;2005(3):238-41

10. Wikler MA. Performance Standards for Antimicrobial Susceptibility Testing: Nineteenth Informational Supplement: Clinical and Laboratory Standards Institute, 2009; M100-S19 CLSI, Wayne, PA

11. Garau J, Xercavins M, Rodriguez-Carballeira M. Emergence and dissemination of quinolone-resistant Escherichia coli in the community. Antimicrob Agents Chemother 1999; 43: 2736-2741

12. Everett MJ, Jin YF, Ricci V, Piddock LJ. Contributions of individual mechanisms to fluoroquinolone resistance in 36 Escherichia coli strains isolated from humans and animals. Antimicrob Agents Chemother 1996;40:2380-2386

13. Livermore DM. Has the era of untreatable infections arrived? J Antimicrob Chemother 2009;64(suppl 1):i29-i36

14. Nema S, Premchandani P, Asolkar MV, Chitnis DS. Emerging bacterial drug resistance in hospital practice. Indian J Med Sciences 1997;51(8):275. 15. Nordmann P, Poirel L. Emergence of plasmid-mediated resistance to quinolones in Enterobacteriaceae. Journal of Antimicrobial Chemotherapy 2005;56(3):463-9

16. Paterson DL. Collateral damage from cephalosporin or quinolone antibiotic therapy. Clinical Infectious Diseases 2004;38(Supple 4):S341-S5 